Gesnerus 64 (2007) 93-108

Essay Review

\title{
L'industrialisation du médicament: une histoire de pratiques entre sciences, techniques, droit et médecine
}

\author{
Jean-Paul Gaudillière
}

Keywords: history of remedies; history of pharmacy; medicine and industry; regulations in science; biotechnology

L'histoire du médicament est un loin d'être un terrain vierge de l'histoire de la médecine. Celle-ci a toutefois longtemps été écrite sur le mode de l'histoire bataille comme une succession d'évènements associant un nom, une date, une découverte de substance et rythmant le progrès des thérapies. Elle a bénéficié, depuis la fin des années soixante-dix, des apports d'une histoire sociale qui a conduit à s'intéresser à la profession de pharmacien, à son statut, aux conditions d'exercice ou de formation. Mais elle est globalement restée une histoire de la science pharmaceutique, des pharmaciens et des substances qu'ils préparent. L'histoire du médicament, en particulier pour le $\mathrm{XX}^{\mathrm{e}}$ siècle, est pourtant de toute évidence aussi une histoire de l'industrie, de ses pratiques de recherche et de production, de la construction des marchés de produits thérapeutiques et des conflits qu'elle a suscitée.

Il y a une dizaine d'années, Michèle Ruffat pouvait écrire: «Si l'histoire de la pharmacie comme profession a fait l'objet de nombreux travaux de recherche historique [...], l'histoire de l'industrie pharmaceutique française, qui est issue de l'officine, a été peu traitée, que ce soit par les historiens économistes ou par les professionnels de cette industrie.» ${ }^{1} \mathrm{Ce}$ constat portait sur l'historiographie française. Les travaux en langue anglaise ou allemande méritent un jugement plus nuancé grâce au développement de la business history et à l'accumulation d'histoires d'entreprises ${ }^{2}$. En regard de l'importance prise au $\mathrm{XX}^{\mathrm{e}}$ siècle par la question du médicament tant dans les pratiques médicales que dans l'économie, le constat d'un relatif désintérêt pour l'histoire de la pharmacie industrielle gardait cependant toute sa pertinence.

1 Ruffat 1995

2 Bäumler 1989; Davenport-Hines/Slinn 1992; Galambos/Sewell 1997; Höllander 1955; Liebenau 1987; Parascandola 1985; Plumpe 1990; Reader 1975; Slinn 1984; Abelshauser 2002.

Jean-Paul Gaudillière, Centre de Recherche Médecine, Sciences, Santé et Société (CERMES), 7, rue Guy Môquet, F-94801 Villejuif Cedex (gaudilli@vjf.cnrs.fr). 
De nombreuses raisons concourent à cette situation. La première est la relative marginalité de l'histoire des techniques et de l'histoire économique dans l'historiographie de la médecine ${ }^{3}$. Une seconde est le fait que l'histoire des sciences a longtemps privilégié la réflexion épistémologique et l'analyse des changements de théorie aux dépens de l'exploration des pratiques matérielles ou des formes sociales d'existence des savoirs ${ }^{4}$. Un dernier facteur est le fait que l'histoire sociale de la médecine s'est surtout intéressée aux représentations collectives de la maladie, à l'organisation des professions médicales, aux dispositifs politiques et sociaux organisant le traitement ou la santé publique 5 .

Depuis une quinzaine d'années, à la faveur d'un contexte plus général de renouvellement des approches de l'histoire du médicament, les travaux sur l'industrie pharmaceutique se sont toutefois diversifiés. Cet essai tente d'en présenter les objets et problématiques en privilégiant l'idée selon laquelle il ne s'agit pas ici d'histoire d'entreprises ou d'histoire d'un secteur économique, mais d'une histoire de l'industrialisation du médicament.

L'industrialisation du médicament au cours du $\mathrm{XX}^{\mathrm{e}}$ siècle a eu pour conséquence une transformation fondamentale de la nature des agents thérapeutiques, des façons de les inventer, de les produire, d'en évaluer les effets et de les commercialiser. Il s'agit d'un phénomène complexe aux dimensions scientifiques, économiques, médicales et politiques que seule une histoire intégrée «des sciences, de la technologie et de la médecine ${ }^{6}$ est susceptible d'éclairer. Dans cette perspective, l'histoire du médicament prend bien évidemment en compte l'industrie comme acteur économique, cherchant par exemple à suivre la constitution d'un secteur du médicament, les formes d'organisation des firmes, leurs relations avec l'Etat ou les assurances, les dispositifs de commercialisation ${ }^{7}$. Elle considère aussi les industries du médicament comme des lieux où l'on produit des savoirs. Cela impose à l'historien de s'intéresser à la nature et aux modalités de la recherche (interne aux entreprises ou menée en coopération), aux formes et stratégies d'appropriation des savoirs, aux relations qu'entretiennent les pratiques de laboratoire et les activités de production. Considérer l'industrie pharmaceutique comme un acteur de la médecine suppose enfin d'explorer les controverses et débats portant sur les effets de telle ou telle spécialité, les formes de collaboration entre firmes, cliniciens et institutions médicales, les dispositifs qui servent à juger de l'efficacité d'un médicament et à définir les normes d'usage.

3 Ce jugement souffre une exception majeure: les études sur l'histoire de l'hôpital, ses formes d'organisation et les technologies qui y sont mobilisées. Sur ce dernier point voir par exemple Howell 1995 et Reiser 1978.

4 Pour une présentation des évolutions de l'historiographie des sciences et plus particulièrement de ses relations à l'histoire des sciences biologiques et médicales, voir Golinski 1998; Bynum 1994; Gaudillière 2006.

5 Faure 1994; Cooter/Pickstone 2000.

6 Pickstone 2000.

7 Il ne s'agit toutefois pas de cette forme d'histoire industrielle dont l'objectif est d'analyser les formes d'organisation et les stratégies d'entreprises pour réfléchir les conditions du succès économique que celui-ci soit défini en termes de revenus, de productivité ou de croissance des entreprises. Pour un exemple récent voir Chandler 2005. 


\section{De l'ordre professionnel à l'ordre industriel}

Le pharmacien exerce une profession purement libérale. C'est lui et lui seul qui est le garant de la qualité des médicaments qu'il délivre, et c'est manquer au devoir professionnel que de substituer aux garanties que présentent les préparations exécutées dans son laboratoire, celles de personnes engagées dans une affaire purement commerciale,

écrivait en 1882 le rapporteur de la commission française de la pharmacopée. Entre ces années 1880 et la Seconde Guerre mondiale, la littérature pharmaceutique est obsédée par le problème des «spécialités», c'est-à-dire des médicaments «prêts à l'emploi» que le pharmacien d'officine n'a plus qu'à stocker et distribuer en fonction des ordonnances qui lui sont présentées. Leur multiplication est la source de multiples conflits révélateurs de remises en cause profondes de l'ordre professionnel de la pharmacie.

Comme pour la médecine, les réformes de la corporation mise en œuvre par les différentes nations européennes au cours du XIX ${ }^{\mathrm{e}}$ siècle dotèrent les pharmaciens d'un statut de profession au sens le plus classique du terme, puisque les pharmaciens jouissaient d'un monopole de fabrication et de vente au nom d'une expertise obtenue par l'admission à un examen organisé par une Faculté ou une Ecole de pharmacie ${ }^{8}$. Les diverses instances (conseils, académies, unions professionnelles ou syndicales) qui l'organisèrent jusqu'au milieu du $\mathrm{XX}^{\mathrm{e}}$ siècle ont poursuivi avec une remarquable constance la défense de ce monopole contre les «charlatans». Mais il ne s'agissait pas seulement de la création d'un corps de praticiens. Les statuts des pharmaciens transformèrent aussi les pharmacopées en Arzneibücher ou en Codex, c'est-à-dire en livres de recettes à valeur légale, fournissant la base de la distinction entre les remèdes que le médecin ordonne de préparer et dont il décrit la composition (préparation magistrale), ceux dont la recette est inscrite dans la pharmacopée (préparation officinale), ceux qui doivent faire l'objet d'une prescription médicale et ceux qui peuvent être délivrés par le pharmacien. En Allemagne, la pharmacopée officielle était établie par un conseil de pharmaciens tandis que la liste des médicaments qui ne pouvaient être délivrés que sur prescription médicale et/ou uniquement par un pharmacien était négociée entre pharmaciens, administration et, à partir de la fin du $\mathrm{XIX}^{\mathrm{e}}$ siècle, représentants de l'industrie'. En France, le Codex était rédigé par une commission de pharmaciens académiques et de médecins désignée par le gouvernement. Le processus d'accord était toutefois suffisamment complexe pour que les révisions n'aient eu lieu qu'à des intervalles de trente à quarante ans $(1818,1837,1866,1884,1908$, 1937). Entre temps, nombre de préparations qui n'étaient pas inscrites au Codex trouvaient leur place dans l'officine, soit parce qu'il s'agissait de «remèdes secrets» à la composition inconnue mais bénéficiant d'une solide réputation, soit parce qu'elles avaient obtenu l'aval des autorités médicales ${ }^{10}$.

L'évolution des statuts, la vie collective de la profession de même que certains aspects de la pratique d'officine ont fait l'objet de travaux conséquents, en particulier ceux d'Olivier Faure en France ou de Erika Hickel en Allemagne. Ce qui est moins connu est le jeu des continuités et discontinuités de pratiques entre le monde de la profession, de l'officine et de la préparation d'une part, et le monde de la spécialité

8 Abbott 1988; Digby 1994; Faure 1989 et 2005; Kibleur 2001; Simon 2005; Wimmer 1994.

9 Wimmer 1994.

10 Faure 1989, 1993 et 1996; Ramsey 1994; Simon 2005. 
industrielle, standardisée, produite en masse et distribuée par des entrepreneurs capitalistes d'autre part.

De nombreuses trajectoires ont ainsi conduit de l'officine familiale à l'entreprise sans solution de continuité. Cette transition ayant surtout été abordée par le biais des histoires d'entreprises, les récits dont nous disposons juxtaposent continuité juridique et économique des compagnies à de grandes discontinuités dans les activités d'invention et de production. Ceci est perceptible dans les biographies de firmes, mais aussi dans des études plus transversales sur la fin du $\mathrm{XIX}^{\mathrm{e}}$ siècle et le début du $\mathrm{XX}^{\mathrm{e}}$ siècle, par exemple celles de Michelle Ruffat sur la France ou de Gabriele Huhle-Kreutzer sur l'Allemagne ${ }^{11}$.

Une des pratiques au cœur de cette transition a été l'industrialisation par la mécanique, à l'arrière-plan de nombreuses innovations galéniques. Une attention croissante accordée au conditionnement a ainsi, selon Anne Rasmussen, changé la préparation sans pour autant passer par l'invention de nouvelles substances et la mise

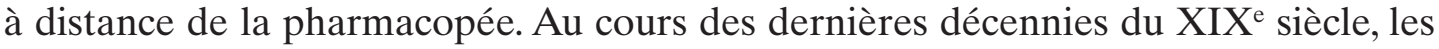
journaux pharmaceutiques comme les foires industrielles exposent les machines à comprimer, à fabriquer les suppositoires, à lyophiliser, à extraire par la vapeur ou les solvants. La spécialité est définie comme un médicament conditionné, c'est-à-dire non seulement comme une substance dont la «présentation» est destinée à optimiser les qualités pharmacologiques mais aussi comme le produit d'un savoir-faire original pour «remplir, compter, peser, mesurer, caler, boucher, étiqueter, marquer, encartonner», lequel serait une garantie tout aussi importante de fiabilité et d'efficacité des produits que la pureté ${ }^{12}$.

Le cas de la firme française Dausse, discuté par Michelle Ruffat, est de ce point de vue intéressant. Dausse avait d'abord été un préparateur d'extraits de plantes. Ses savoir-faire portaient moins sur les technologies de purification que sur les procédures de manipulation des végétaux. Le point de départ est l'invention d'appareils permettant d'accomplir plus rapidement ces réactions de déplacements qui, au début du XIX ${ }^{\mathrm{e}}$ siècle, sont tout aussi importantes à la préparation des extraits actifs que les procédés d'analyse. Les innovations de Dausse ne relèvent donc pas de l'apparition de nouvelles substances. Les produits exploités sont connus depuis longtemps et inscrits dans le Codex: valériane, gentiane, belladone, colchique, digitale, strophantus, gui, marron d'inde, etc. Les points forts de la compagnie sont la variation des modes de présentation, la stabilité des combinaisons offertes aux praticiens. Dausse domine l'utilisation des plantes au début du $\mathrm{XX}^{\mathrm{e}}$ siècle, lorsque la firme combine l'amélioration des techniques de traitement des plantes par la vapeur ou par le vide avec des innovations organisationnelles comme l'abandon de la collecte, le passage à la plantation et à la culture directe des plantes médicinales.

Les rapports entre pharmacie et production industrielle de médicament ne sont pas que d'empathie. La multiplication du nombre des spécialités (plusieurs dizaines de milliers occupent les marchés de l'Europe industrialisée dans l'entre-deux-guerres) suscitent une forte hostilité des unions de pharmaciens ${ }^{13}$. A la fin des années trente, la bible de la Faculté de Pharmacie de Paris, le Traité de Thérapeutique et de Pharmacologie, rédigée par G. Hazard proclamait:

11 Ruffat 1996; Huhle-Kreutzer 1989.

12 Rasmussen, A. 2004 et 2005.

13 Chauveau 1999; Wimmer 1994. 
Quelques unes d'entre elles [les spécialités], par leur mode de préparation, exigent des laboratoires spécialement outillés et leur utilité est hors de discussion. La plupart des autres représentent soit des produits qui n'ont pas d'autre caractère de pureté que ceux du Codex, soit des mélanges de médicaments dont l'association répond à des conceptions thérapeutiques parfois discutables. Beaucoup d'entre elles pourraient disparaître sans dommage pour le malade. Leur succès a des causes complexes: leur présentation agréable est peut-être la principale. On dit aussi que le malade est assuré de les trouver partout, toujours semblables à elles-mêmes. [...] A la vérité le succès de la plupart des spécialités tire sa source, il faut le dire, de la négligence qu'apportent beaucoup de médecins à s'instruire dans l'art de formuler. ${ }^{14}$

Ces tensions ont débouché sur des tentatives pour renforcer le monopole de vente, maintenir la part des préparations et surtout limiter les mises sur le marché. Le débat allemand de la fin du XIX ${ }^{\mathrm{e}}$ siècle sur la nature, les dangers possibles et les limites à poser à la mise en circulation des comprimés en est un exemple ${ }^{15}$. Souvent conduites au nom de la santé publique et de la protection contre les effets toxiques, ces tentatives ont joué un rôle non négligeable dans la transformation des formes d'encadrement de l'activité pharmaceutique, débouchant dans certains cas sur un renforcement des contrôles et de la surveillance étatique.

Instauré par le régime de Vichy, dans un contexte qui combinait renforcement du rôle des élites corporatives et revendication technocratique de la modernité industrielle, le système français du visa est un exemple de ces compromis établis autour de la gestion de la mise sur le marché ${ }^{16}$. Le visa était un dispositif propre aux spécialités industrielles par lequel l'Etat, via un Comité technique des Spécialités (CTS) auprès de la Direction de la Pharmacie, jugerait de l'intérêt d'une nouveauté. La composition du comité était l'expression d'une nouvelle forme d'expertise qu'on peut dire «paritaire» puisqu'on y retrouvait pharmaciens, administrateurs et représentants de l'industrie. L'attribution d'un visa de commercialisation devait être précédée d'une évaluation de la non-toxicité et des propriétés thérapeutiques du médicament (enjeu de santé publique), de la conformité aux compositions annoncées (lutte contre les remèdes secrets et l'automédication), du caractère innovant (substitut de propriété intellectuelle). Dans les formulations de la loi, le CTS devait s'assurer que le produit ne nuisait pas à la santé ou à la moralité publique; n'était pas dépourvu de toute activité thérapeutique; avait une formule correctement établie; répondait à la formule annoncée ${ }^{17}$. Le fabricant restait maître de la nature des essais à pratiquer. Leur nature devait cependant être précisée dans la demande d'homologation et approuvée par les membres du CTS. Ce dernier pouvait servir d'intermédiaire entre le fabricant et les experts susceptibles de l'aider à préparer sa demande et rémunérés par lui. Conçu comme une incitation à l'innovation de composition ou d'indication, le visa était associé à un autre dispositif, dit des «produits sous cachet». Ce dernier permettait la vente, sous le nom d'un pharmacien, de produits de composition déjà connue car définie par le Codex ou identique à celle d'une spécialité ayant fait l'objet d'un visa. Ce système visait à préserver le droit à la préparation des pharmaciens. Le CTS fut vite débordé par les demandes. S'estimant peu compétent pour juger du fond, il eut essentiellement pour rôle d'aider les fabricants à trouver les «bons» experts,

14 Hazard 1938.

15 Wimmer 1994.

16 Chauveau 1999 et 2005.

17 Décret-loi du 11 septembre 1941. 
ceux dont l'avis convaincrait. Le visa fonctionna donc surtout comme une chambre d'enregistrement, organisant le transfert vers les industriels et vers l'administration d'une partie des prérogatives de la profession, en particulier l'expertise de la nontoxicité et de l'intérêt thérapeutique des produits.

\section{Recherche industrielle: la saga chimique revisitée}

La transformation de la préparation thérapeutique en entité standard, produite en masse et facilement transportable a le plus souvent été associée au contexte de la seconde révolution industrielle, en particulier à l'entrée sur le marché du médicament des grandes entreprises de la chimie comprise comme le point de départ d'une modernisation entendue comme une «scientificisation» de l'invention basée sur la mobilisation systématique du laboratoire. Cette modernisation est aussi une «molécularisation» du médicament dans la mesure où la quête de composés thérapeutiques homogènes, contrôlés, agissant de façon spécifique sur l'agent ou le processus tenu pour responsable d'une pathologie, a privilégié la purification et la synthèse des substances. Le point d'aboutissement en a été la technique du criblage qui associe la genèse de milliers de molécules analogues et l'examen systématique de leur potentiel thérapeutique dans des essais biologiques standardisés. L'histoire des usages thérapeutiques des colorants est l'exemple le plus souvent cité de cette industrialisation par la chimie organique ${ }^{18}$.

Entre 1860 et 1880, après l'isolement du dérivé mauve de l'aniline par le chimiste anglais W. Perkin, la synthèse de dizaines de colorants possédant la même structure de base a radicalement transformé les pratiques de la chimie et de l'industrie textile ${ }^{19}$. L'élaboration de ces composés a fourni un stock de réactions à partir desquelles les chimistes «organiciens» ont élaboré les théories sur la structure des noyaux aromatiques et des groupements latéraux. Elle a changé les relations entre universitaires et industriels, suscitant aussi bien la création de firmes destinées à exploiter les protocoles que des chimistes comme Friedrich Bayer avaient inventé, que la mise en place, au sein même des compagnies, de laboratoires chargés de surveiller la production et de trouver des molécules nouvelles. Les colorants ont finalement fait la fortune de quelques firmes: Bayer, Hoechst, BASF ensuite intégrées au sein du trust IG Farben, mais aussi la Société des Usines du Rhône en France ou Imperial Chemical Industries en Grande-Bretagne ${ }^{20}$.

La production des colorants a débouché sur celle de substances thérapeutiques selon deux voies différentes. La première a été l'exploitation directe de la synthèse organique pour modifier les conditions de production des médicaments connus comme l'acide dérivé de l'écorce de saule dont Bayer fit l'aspirine en 1899. La seconde voie a été l'utilisation de certaines substances colorantes au laboratoire de bactériologie. L'emploi le plus immédiat était la coloration des préparations microscopiques au moyen de fuschine, de bleu de méthylène, de vert crésol, etc. Le raffinement des protocoles de visualisation en a fait des outils de diagnostic différentiel, étayant l'idée selon laquelle les bactéries - tout comme les fibres textiles - présentent, en fonction

18 Chast 1995; Liebenau 1990; Weatherall 1990.

19 Bäumler 1989; Travis 1993.

20 Cayez 1996; Fischer 1991; Homburg 1992; Lesch 2000; Plumpe 1990; Reader 1975. 
de la structure chimique de leur surface, des affinités électives pour certaines matières colorantes. Sur cette base, des chercheurs comme Paul Ehrlich ont cherché à exploiter les procédés de la synthèse organique pour trouver des molécules se fixant spécifiquement sur un germe pathogène et doté d'une action toxique ${ }^{21}$.

Le rêve d'une «chimiothérapie» s'est d'abord concrétisé par la mise au point du Salvarsan ${ }^{22}$. S'intéressant à la syphilis après l'isolement de son agent en 1905, Ehrlich examina une importante série de dérivés contenant de l'arsenic, cherchant à obtenir un équilibre entre toxicité pour l'organisme et pouvoir de destruction du tréponème. Cette exploration introduisait une nouvelle façon de chercher, dans la mesure où elle associait la fabrication ou la collecte des substances à leur test sur les cultures bactériennes et sur des lapins infectés. Le succès du modèle doit beaucoup au fait que même s'il était encore très toxique, le composé numéro 606 mis en vente à partir de 1910 par Hoechst prenait pour cible un «fléau». Ehrlich passa les cinq années suivantes à répondre aux critiques sur les effets variables et la faible tolérance à son produit, multipliant les collaborations avec les cliniciens pour augmenter les doses, limiter les réactions allergiques et empêcher les rechutes. La réponse chimique au problème clinique fut la synthèse d'une arsephanime modifiée, le Néo-Salvarsan, commercialisé en 1915.

L'idée selon laquelle c'est la systématisation et l'internalisation de ce paradigme pour constituer un modèle d'organisation industrielle de recherche qui aurait conduit à la révolution thérapeutique d'après la Seconde Guerre mondiale est habituellement discutée en relation avec l'histoire des sulfamides ${ }^{23}$. Celle-ci a été réévaluée sur plusieurs points essentiels.

Analysant le cas de Bayer, John Lesch a insisté sur l'articulation entre les activités de synthèse élargie de molécules du laboratoire de chimie et la pratique systématique des tests bactériologiques sélectionnés par les pathologistes embauchés par la firme ${ }^{24}$. Dans cette perspective, le screening n'est pas une activité empirique, mais un système construit sur les allers et retours entre deux types de savoirs. Il s'agit d'un jeu entre ce que l'on sait chimiquement faire et ce qui est biologiquement intéressant, dominé par l'idée selon laquelle l'action thérapeutique dépend uniquement de la nature des structures de base et des radicaux que l'on peut greffer sur celles-ci. La structure même du Prontosil, premier sulfamide mis sur le marché en 1935, incarnait cette combinaison avec un noyau central issu de la lignée des colorants et une chaîne latérale sélectionnée au laboratoire de pharmacologie. Mais on a maintes fois souligné ce paradoxe qui veut que, quelques mois après la publication princeps des résultats de Bayer, les chercheurs de l'Institut Pasteur montrèrent que la partie colorante n'était pour rien dans l'action anti-bactérienne, déplaçant la cible du screening vers le groupement sulfonamide.

Suivant la diversification des sulfamides au-delà des activités de Bayer, Lesch montre l'importance des réseaux de collaboration externe des firmes, en particulier avec un petit nombre de cliniciens, souvent des collaborateurs de longue durée, impliqués dans la spécification pharmacologique des substances (examen de la toxicité, définition des relations entres doses et effets) et dans la recherche des indications. Le cas

21 Prüll 2003; Travis 1989.

22 Bäumler 1979 et 1989; Liebenau 1990.

23 Bickel 1988; Bovet 1988; Chast 1995; Weatherall 1990.

24 Lesch 1993 et 2007. 
de la firme britannique May \& Backer, succursale de Rhône-Poulenc, illustre le rôle essentiel de cette organisation de la clinique et de ses retours d'expérience pour la mise au point et la stabilisation des premières thérapies chimiques de la pneumonie.

Le cas des psychotropes montre lui aussi comment des molécules qui avaient été sélectionnées pour une certaine catégorie d'usage dans les laboratoires internes de Rhône-Poulenc ont été, dans les années cinquante, totalement redéfinies par la clinique $^{25}$. Le premier neuroleptique, le Largactyl, faisait ainsi partie d'une famille de molécules examinées par les chercheurs de Rhône-Poulenc pour leur activité antihistaminique. Remis dans la bibliothèque de molécule de la firme pour cause d'effet médiocre, il fut confié au physiologiste Henri Laborit qui en fit un médicament sédatif et un outil de recherche sur le problème du choc. Il ne devint un anti-psychotique que lorsque les psychiatres de l'hôpital Sainte-Anne à Paris s'en saisirent. Initialement commercialisé comme traitement symptomatique de l'asthme, des vomissements ou des insomnies, le Largactyl accèda alors au rang de traitement spécifique de la psychose. Cette association entre caractérisation moléculaire et redécoupage des frontières de la pathologie est à l'origine du succès des neuroleptiques. Le cas est loin d'être unique. Invention clinique, définition tardive de la cible scientifique et recomposition des diagnostics en fonction de l'utilisation des molécules se retrouvent dans les trajectoires de la plupart des psychotropes ${ }^{26}$.

D'une façon plus générale, à partir de l'entre-deux-guerres, les plus grandes compagnies créent d'une part des sections de bactériologie, parfois de physiologie, consacrées à la modélisation des effets des substances et d'autre part des départements médicaux. Les travaux des premières viennent relayer, stimuler ou entrer en compétition avec ceux decollaborateurs académiques qui avaient auparavant constitué la principale source d'expertise biologique et pharmacologique ${ }^{27}$. Les seconds assument un double rôle. La première fonction de ces départements médicaux était de «faire remonter» une partie de l'expérience clinique tout en laissant - semble-t-il - une assez grande marge de manœuvre aux praticiens dans l'appréciation des usages et des essais, les départements médicaux se chargeant de l'agrégation des cas $^{28}$. La seconde fonction était le marketing et la construction des marchés. A partir des années trente, les plus grandes firmes organisent périodiquement séminaires et réunions «médicales», lancent des journaux qui juxtaposent publicités et articles de recherche reproduits de la presse médicale ou émanant de leurs propres laboratoires. Ces activités de «publicité scientifique» restent à étudier, mais on peut penser qu'elles ne sont devenues déterminantes dans la définition des usages des médicaments que dans l'après-guerre avec l'organisation du système des visiteurs. Le cas des compagnies américaines montre comment la systématisation des visites a pris appui sur les techniques de recherche marketing pour constituer un dispositif (contesté) d'enregistrement, de discussion et de formatage des prescriptions ${ }^{29}$.

La diversité des pratiques de recherche faussement attribuées à la chimie industrielle reste à explorer, en particulier pour ces médicaments biologiques qui constituent, à partir des années 1920, une part importante des médicaments industriels.

25 Bonah/Rasmussen 2005; Swazey 1974.

26 Healy 2002.

27 Gaudillière 2004 et 2005; Quirke 1999; Rasmussen, N. 2004; Swann 1988.

28 Rasmussen, N. 2004 et 2005.

29 Greene 2004. 
Leur production prolonge la culture de la préparation, mais privilégie standardisation, élaboration en masse et productivité. Vaccins, hormones sexuelles, corticostéroïdes et antibiotiques sont ceux qui ont jusqu'à présent (inégalement) retenu l'attention ${ }^{30}$.

L'historiographie du plus célèbre de ces «biologiques» - la pénicilline - montre tout l'intérêt d'une exploration des «trajectoires» des médicaments. On sait que l'invention de la pénicilline commence par une «pure» découverte de laboratoire faite par Alexander Fleming, microbiologiste au St Mary's Hospital de Londres où il travaillait sur la vaccinothérapie ${ }^{31}$. Pourtant, Fleming n'a pas découvert la pénicilline, puisque «sa» pénicilline n'était pas un médicament. Déçu par ses premiers essais pour utiliser le bouillon de culture de Penicillium comme antiseptique externe, convaincu qu'une chimiothérapie interne était un rêve inaccessible, Fleming l'employa comme réactif pour obtenir des cultures pures de bactéries dont il pensait qu'elles étaient responsables de la grippe ${ }^{32}$. Une seconde pénicilline apparaît dix ans plus tard lorsque des biochimistes de l'Université d'Oxford, dirigés par Howard Florey, purifient une substance active contre les staphylocoques et les streptocoques parce qu'ils pensent qu'elle fait partie d'une famille de protéines aux propriétés anti-bactériennes $^{33}$. Urgence militaire aidant, au début des années quarante, ils tentent d'en faire un médicament, traitent des souris et quelques patients, les premières avec succès, les seconds avec moins de bonheur. La pénicilline efficace est le produit d'une troisième rupture. Convaincus que leurs échecs tiennent à l'insuffisance des doses, Florey et un de ses assistants traversent l'Atlantique à l'été 1941 pour démarcher les compagnies américaines. En vain, car celles-ci misaient en priorité sur la diversification des sulfamides. Le programme «pénicilline» est la création d'une administration publique, l'Office for Scientific Research and Development (OSRD), en charge des recherches de guerre. Investissant dans la quête de souches plus productives du champignon, dans la mise au point des conditions de culture, dans les techniques de fermentation en profondeur dans des grands conteneurs, l'OSRD négocia la mise à disposition de ses connaissances, convainquant les compagnies de s'associer à la production de l'antibiotique $^{34}$. L'office soutint un vaste programme de recherche pour arriver à une synthèse chimique complète. C'est ce pari techno-industriel qui a finalement fait de la pénicilline un composé disponible en masse et efficace en clinique ${ }^{35}$. La dernière ironie de l'histoire est que la pénicilline est restée un produit biologique, produit par fermentation, le procédé de synthèse finalisé en 1947 se révélant trop coûteux. Ce n'est donc que dans les années cinquante que l'histoire des antibiotiques rejoint celle du screening moléculaire avec la multiplication des analogues des antibiotiques naturels, désormais considérés non seulement comme moyen de diversifier l'arsenal thérapeutique mais aussi pour contourner le problème récurrent des résistances à la pénicilline ${ }^{36}$.

30 Galambos/Sewell 1997; Gaudillière 2004; Hess, à paraître; Marks, H. 1992; Marks, L. 2001; Oudshoorn 1994.

31 Macfarlane 1984.

32 Chen 1996.

33 Macfarlane 1979.

34 Neushul 1993.

35 Hobby 1985.

36 Bud 2007. 
Suivre les transformations d'un médicament au cours des différentes phases de son «développement» est une idée aussi ancienne que l'histoire de la pharmacie, parfois associée au terme de «biographie». La notion de trajectoire vise au contraire à insister non pas sur la continuité et la stabilité de l'agent thérapeutique, mais sur les processus de sa construction comme substance matérielle et comme outil thérapeutique. C'est en particulier une manière d'insister sur les circulations entre lieux de science, de production et de soin. La perspective est double. Il s'agit d'une part de comprendre comment les différents types de pratiques entrent en interaction, comment l'efficacité est un produit tardif et non la cause des choix et processus; et d'autre part de prendre en compte le travail d'établissement des frontières, la réalité des tensions entre laboratoire et usine, entre entreprise et hôpital, entre cabinet et officine.

Outre les antibactériens - chimiques ou biologiques -, ce sont des médicaments comme les psychotropes évoqués plus haut, les corticostéroïdes, les anticancéreux, les sérums antidiphtériques, les hormones, en particulier sexuelles qui ont fait l'objet de ce type d'approche ${ }^{37}$. L'histoire de l'insuline a ainsi été écrite par Michael Bliss du seul point de vue du laboratoire ${ }^{38}$. Christiane Sinding a récemment analysé l'ensemble des collaborations qui ont fait de l'insuline un médicament standard et efficace. Cela lui a permis de poser la question de la standardisation en termes d'interaction et non pas de stabilisation des caractéristiques techniques d'un essai. Elle souligne non seulement le rôle des physiologistes et de leurs modèles, mais aussi l'existence d'une «standardisation clinique» faisant de l'unité d'insuline une entité définie par la pratique thérapeutique. De plus, les informations sur les réactions des patients et les effets secondaires fournies à Eli Lilly par les médecins prescripteurs ont non seulement permis la mise au point des posologies et des protocoles d'administration, mais aussi la standardisation des procédés de fabrication de l'hormone ${ }^{39}$.

\section{Une marchandise comme les autres? Brevet, statut et régulation du médicament industriel}

Faut-il breveter les médicaments? Sûrement pas, ont répondu pendant près de cent cinquante ans pharmaciens et autorités sanitaires de la plupart des pays européens ${ }^{40}$. Abcès de fixation des conflits entre logiques de la production marchande et santé publique, les discussions sur la brevetabilité des agents thérapeutiques ont été récurrentes depuis le milieu du XIX ${ }^{\mathrm{e}}$ siècle. D'un côté, on trouvait les chimistes et les firmes en voie d'industrialisation arguant de la qualité nouvelle des remèdes, de leur pureté et de la constance de leurs propriétés garanties par la science chimique et l'invention industrielle. En face, les défenseurs de la pharmacie d'officine insistaient sur l'accès indispensable aux modes de préparation, sur la protection contre les fraudes et la mauvaise qualité et surtout sur les effets néfastes du monopole garanti par le brevet sur les prix, l'accès aux spécialités et, par voie de conséquence, la santé de la population.

37 Gaudillière 2004 et 2005; Goodmann/Walsh 2001; Hess, à paraître; Oudshoorn 1994; Quirke 2004 et 2005; Rasmussen, N. 2004.

38 Bliss 1984.

39 Sinding 2002.

40 Cassier 2004 et 2005; Chauveau 1999; Dutfield 2003; Fleischer 1984; Tanner 1998; Wiemmer 1994. 
Les choses ont radicalement changé après la Seconde Guerre mondiale. En l'espace d'une quinzaine d'années, les grands pays producteurs se sont dotés d'une législation autorisant les brevets de substances thérapeutiques (Grande-Bretagne 1949, France 1959, Allemagne 1965). Quelles sont les racines de cette évolution?

Une première explication, privilégiée par l'histoire économique, met en avant l'industrialisation de la production qui a rapproché le médicament des autres marchandises, les entreprises de ce secteur des autres firmes capitalistes. La brevetabilité n'a fait qu'enregistrer une «normalisation» du marché de la santé portée par la croissance des entreprises, les changements de la production, les investissements dans la recherche interne, les nouvelles stratégies de marketing. L'évolution des systèmes de santé après 1945 a aussi contribué à miner les résistances. La généralisation de la couverture maladie a assuré une croissance rapide de la demande et une garantie de solvabilité relativisant la critique du monopole créé par le brevet.

Un second registre d'explication, plus récent, fait écho à l'histoire du droit et insiste sur les processus de construction du statut marchand des agents thérapeutiques, en particulier les modalités de gestion de la propriété intellectuelle au sein des entreprises, des laboratoires, des offices des brevets ${ }^{41}$. En dépit des exclusions formelles, les procédés de préparation des médicaments pouvaient en effet faire l'objet de multiples protections: par le secret, par le dépôt d'une marque déposée, par la possession d'un monopole de technologie et de savoir-faire. Plus, à partir du début du $\mathrm{XX}^{\mathrm{e}}$ siècle, non seulement les procédés mécaniques (les machines à fabriquer les comprimés), mais aussi les procédés chimiques peuvent faire l'objet d'un brevet. Une question décisive était ainsi celle des pratiques juridiques, de la façon dont la frontière entre brevet de procédé et brevet de médicament était définie. En Allemagne, à partir de la fin du XIX ${ }^{\mathrm{e}}$ siècle, se constitue ainsi autour des grandes firmes un véritable «milieu» du brevet associant les juristes des firmes, les ingénieurs de production, certains universitaires, les fonctionnaires de l'office des brevets. S'appuyant sur cette expertise, les juristes ont progressivement étendu la protection par brevet d'abord au contrôle des procédés de fabrication des médicaments, ensuite aux procédés de préparation des substances biologiques à partir du moment où la spécificité des protocoles était garantie par l'existence d'un essai biologique standard. En France, le recours au brevet de procédé a été moins systématique, la jurisprudence plus contradictoire mais des déplacements analogues se font jour dès les années trente. En Angleterre, par contre, il semble que l'«économie morale» d'un monde universitaire hostile aux entreprises commerciales ait davantage pesé, renforçant les réticences des médecins et conduisant, par exemple, à une politique de non brevetabilité du Medical Research Council fortement critiquée, après 1945, lorsque le sort de la pénicilline, dont les procédés de fabrication furent en partie breveté aux Etats-Unis, devint une affaire nationale.

Les compromis entre propriété et santé publique n'ont pas disparu avec la normalisation d'après-guerre. Le premier système établi en France était un «Brevet Spécial du Médicament» (BSM) intégré au code de la santé publique ${ }^{42}$. Dans ce cadre, l'Etat se réservait le droit de limiter le périmètre et les conditions d'usage des brevets accordés par l'Institut National de la Propriété Industrielle. Pour contrer les possibilités de monopole ou d'abus de position dominante, il pouvait imposer des licences

41 A ce propos voir le numéro spécial History and Technology, «Patenting Drugs» à paraître. 42 Chauveau 1999. 
obligatoires au profit de tiers. D'autre part, si l'inventeur n'en faisait aucune exploitation, le brevet pouvait être annulé. En 1968, sous la pression du Ministère de l'Economie et en lien avec les exigences de construction de la communauté économique européenne, le BSM fut transformé en brevet usuel, au nom du fait que le dispositif de la licence obligatoire n'avait jamais été utilisé.

Un autre changement d'importance de l'après-guerre auquel l'historiographie des pratiques s'est récemment intéressée est le renforcement de la régulation, c'est-à-dire des dispositifs mis en œuvre pour évaluer les effets, la toxicité et l'efficacité d'un agent thérapeutique avant sa mise en vente ou sa mise à disposition. D'abord affaire de la profession, cette régulation est devenue, dans le contexte de l'industrialisation, un problème de contrôle de qualité dont la gestion a été marquée par l'introduction, au sein des entreprises ou, comme dans le cas du contrôle allemand des sérums, au sein de laboratoires publics de services de tests et d'essais de toxicité des médicaments industriels. Durant la seconde moitié du XX ${ }^{\mathrm{e}}$ siècle, le modèle des agences dites de «régulation», spécialement créées à cet effet par l'Etat, s'est généralisé. Surtout ces agences sont devenues responsable de l'examen de l'ensemble des propriétés du médicament (composition, toxicité, efficacité) et des autorisations de mise sur le marché.

L'histoire de la Food and Drug Administration (FDA) américaine est le cas le plus discuté pour analyser ce processus. La FDA a été créée en 1905 pour lutter contre l'adultération des aliments et des médicaments ${ }^{43}$. Elle n'a acquis le pouvoir de décider si un médicament est toxique et s'il est efficace qu'à travers les réformes législatives de 1938 et 1962 qui ont imposé aux fabricants de fournir la preuve de leurs annonces. Ces réformes sont le produit non pas de «scandales» ayant donné aux autorités politiques la légitimité pour intervenir et renforcer le contrôle des marchés, mais de situations de crise complexe qui ont modifié le jeu des alliances, intérêts et pouvoirs respectifs des professionnels de la médecine, des industriels, des usagers et des autorités de santé publique. En 1938, l'événement catalyseur a été le décès de plusieurs dizaines de patients dû à la mise sur le marché par la firme $\mathrm{S}$. E. Massengill d'une préparation de sulfanilamide additionnée de diéthylène glycol. L'affaire qui en résulta permit, dans le contexte du New Deal, à une partie de l'élite médicale méfiante vis-à-vis des pratiques de l'industrie et préoccupée par la «crédulité» de leurs collègues de faire alliance avec les responsables de l'agence et du Public Health Service pour imposer et normaliser les tests de toxicité ${ }^{44}$. En 1962, le point de départ fut une catastrophe potentielle et un scandale réel. La prescription de thalidomide, un somnifère largement employé parce que ne provoquant pas de nausées ou de somnolence le lendemain de son utilisation, est à l'origine de la naissance, en Europe, de plusieurs milliers de nouveaux-nés affectés par des malformations. Au moment où l'affaire éclata, la FDA était en train d'examiner le dossier d'homologation. La mise en cause du système d'autorisation qui avait permis au producteur américain de distribuer la thalidomide à titre expérimental déboucha sur un renforcement considérable des pouvoirs de la FDA. Méfiants vis-à-vis d'une industrie que sa croissance avait rendue trop puissante, les membres du Congrès se rangèrent à l'avis des cliniciens réformateurs et imposèrent l'organisation d'essais contrôlés de l'efficacité comme condition préalable à l'examen des demandes de mise sur le marché ${ }^{45}$.

43 Young 1967 et 1989.

44 Jackson 1970; Marks, H. 1997.

45 Daemmerich 2004; Marks, H. 1997. 
La régulation étatique ne s'est pas imposée partout de la même manière. Comparant les discussions sur la thalidomide, les sulfamides et l'interleukine-2 aux EtatsUnis et en Allemagne, le sociologue Arthur Daemmerich insiste les différences entre «cultures thérapeutiques» nationales. Il oppose une configuration américaine où le jugement des thérapies est public, conflictuel, mobilise les médias et les organisations de la société civile qui font pression pour que l'agence étatique intervienne, à une configuration allemande où la délégation d'expertise aux instances professionnelles qui négocient avec l'industrie est resté la forme dominante de gestion des médicaments. Le cas de la thalidomide est là encore pris en exemple. En Allemagne, l'affaire se régla par la négociation entre l'Etat et la firme productrice, avec la création d'un fonds d'indemnisation. La réforme vint quelques années plus tard, sous la forme d'un système d'enregistrement comparable au visa français et reposant sur l'association entre professionnels et entreprises du médicament. L'essai contrôle et randomisé n'y devint norme légale que dans les années quatre-vingt, dans le cadre de l'unification européenne des statuts dont le dernier épisode a été la création d'une agence commune coiffant les institutions nationales.

Pour conclure cette discussion, on insistera sur le fait que cette historiographie des agents thérapeutiques privilégiant l'étude des pratiques scientifiques, techniques, juridiques et médicales n'a pas seulement introduit de nouveaux objets et de nouvelles approches. Elle fait écho et contribue à éclairer nombre des enjeux de notre présente pharmaceutique, de sorte qu'elle pourrait constituer la base d'un dialogue plus soutenu avec les études du contemporain. Ceci est évident si l'on considère les controverses contemporaines sur le brevet, la propriété intellectuelle, les questions d'accès au médicament, en particulier dans les pays du Sud. Mais cela est aussi vrai de questions moins directement liées au fait que les médicaments ne sont, malgré leur industrialisation, jamais devenus des marchandises ordinaires. Il en est ainsi de cette «crise de l'innovation» pointée par de nombre d'observateurs et qui se manifeste par la réduction du nombre de mises sur le marché de molécules innovantes, la multiplication des médicaments me-too, le durcissement des comportements propriétaires $^{46}$. Les racines de cette crise tiennent sans doute pour partie aux évolutions des régulations économiques et juridiques générales, qu'il s'agisse des transformations des rapports entre recherche publique et recherche privée ou du développement des marchés financiers. Mais elles renvoient aussi à l'épuisement du modèle d'innovation centré sur les pratiques du screening moléculaire qui s'est stabilisé et généralisé durant la seconde moitié du siècle et que le recours aux biotechnologies génétiques n'a pas (encore?) suffi à renouveler.

\section{Bibliographie}

Abbott,Andrew, The System of Professions: An Essay on the Division of Expert Labour (Chicago 1988)

Abelshauser, Werner (Hrsg.), Die BASF. Eine Unternehmensgeschichte (München 2002)

Bäumler, Ernst, Paul Ehrlich. Forscher für das Leben (Frankfurt a.M. 1979)

- Farben, Formeln, Forscher. Hoechst und die Geschichte der industriellen Chemie in Deutschland (München 1989)

Bickel, Marcel, "The development of Sulfonamides (1932-1938) as a focal point in the history of chemotherapy", Gesnerus 45 (1988) 67-86

46 Goozner 2004; Pignarre 2003; Urfalino 2005. 
Bliss, Michael, The Discovery of Insulin (Chicago 1984)

Bonah, Christian/Anne Rasmussen (éds), Histoire et médicament aux $19^{e}$ et $20^{e}$ siècles (Strasbourg 2005)

Bovet, Daniel, Une chimie qui guérit: histoire de la découverte des sulfamides (Paris 1988)

Bud, Robert, Penicillin. Triumph and Tragedy (Oxford 2007)

Bynum, William, Science and the Practice of Medicine in the 19th Century (Cambridge 1994)

Cassier, Maurice, «Brevets pharmaceutiques et santé publique en France: opposition et dispositifs spécifiques d'appropriation des médicaments entre 1791 et 2004», Entreprise et Histoire 36 (2004) 29-46

- "Appropriation and commercialization of the Pasteur Anthrax vaccine", Studies in History and Philosophy of Biological and Biomedical Sciences 36 (2005) 722-742

Cayez,Pierre, Rhône-Poulenc 1875-1975: Contribution à l'étude d'un groupe industriel (Paris 1996)

Chandler, Alfred, Shaping the Industrial Century. The Remarkable Story of the Evolution of the Modern Chemical and Pharmaceutical Industries (Cambridge M.A. 2005)

Chast, François, Histoire contemporaine des médicaments (Paris 1995)

Chauveau, Sophie, L'invention pharmaceutique. La pharmacie française entre l'Etat et la société au $20^{e}$ siècle (Paris 1999)

- «Le statut légal du médicament», dans: Bonah/Rasmussen (éds), Histoire et médicament au $19^{e}$ et $20^{e}$ siècles (Strasbourg 2005)

Chen, Wai, Comment Fleming n'a pas inventé la pénicilline (Paris 1996)

Daemmerich, Arthur, Pharmacopolitics: Drug Regulation in the United States and Germany (Chapel Hill 2004)

Cooter, Roger/John Pickstone (eds), Medicine in the 20th Century (Amsterdam 2000)

Davenport-Hines, Richard/Judy Slinn, Glaxo: A History to 1962 (Cambridge 1992)

Digby, Anne, Making a Medical Living: Doctors and Patients in the English Market for Medicine (1720-1911) (Cambridge 1994)

Dutfield, Graham, Intellectual Property Rights and the Life Science Industries: A Twentieth Century History (Aldershot 2003)

Faure, Olivier, «Officines, pharmaciens et médicaments en France au $19^{\mathrm{e}}$ siècle», Bulletin de la Société d'histoire moderne et contemporaine 44 (1989) 31-39

- Les Français et leur médecine au $19^{e}$ siècle (Paris 1993)

- Histoire sociale de la médecine (Paris 1994)

- «Les officines pharmaceutiques françaises: de la réalité au mythe (fin XIX ${ }^{\mathrm{e}}$-début XX ${ }^{\mathrm{e}}$ siècle)», Revue d'Histoire Moderne et Contemporaine 43 (1996) 672-685

- «Les pharmaciens et le médicament en France au XIX ${ }^{\mathrm{e}}$ siècle», dans: Bonah, Christian/Anne Rasmussen (éds), Histoire et médicament aux $19^{e}$ et $20^{e}$ siècles (Strasbourg 2005) 65-85

Fischer, Ernst P., Wissenschaft für den Markt. Die Geschichte eines forschenden Unternehmens Boehringer Mannheim (Zürich 1991)

Fleischer, Arndt, Patentgesetzgebung und chemisch-pharmazeutische Industrie im deutschen Kaiserreich (1871-1918) (Stuttgart 1984)

Galambos, Louis/Jane Sewell, Networks of Innovation: Vaccine Development at Merck, Sharp and Dohme and Mullford, 1895-1995 (Cambridge 1997)

Gaudillière, Jean-Paul, «Hormones, régime d'innovation et stratégies d'entreprise: les exemples de Schering et Bayer», Entreprise et Histoire 36 (2004) 84-102

- "Introduction: Drug Trajectories", Studies in History and Philosophy of Biological and Biomedical Sciences 36 (2005) 603-611

- "Better prepared than synthesized: Adolf Butenandt, Schering AG and the transformation of sex steroids into drugs", Studies in History and Philosophy of Biological and Biomedical Sciences 36 (2005) 612-644

- La médecine et les sciences. XIX $X^{e}-X X^{e}$ siècles (Paris 2006)

Golinksi, Jan, Making Natural Knowledge: Constructivism and the History of Science (Cambridge 1998)

Goodmann, Jordan/Vivian Walsh, The Story of Taxol (Cambridge 2001)

Goozner, Merrill, The 800 Million Dollar Pill. The truth behind the cost of new drugs (Berkeley 2004)

Greene, Jeremy, "Attention to details: etiquettes and the pharmaceutical salesman in postwar America”, Social Studies of Science 34 (2004) 271-292 
Hazard, G., Traité de Thérapeutique et de Pharmacologie (Paris 1938)

Healy, David, The Creation of Psychopharmacology (Cambridge M.A. 2002)

Hess, Volker, "The administrative stabilization of vaccines: regulating the diphteria antitoxin in France and Germany, 1894-1900", Science in context, à paraître

Hobby, Gladys, Penicillin: Meeting the Challenge (New Haven 1985)

Höllander, Hans, Geschichte der Scheuny-AG (Berlin 1955)

Homburg, E., "The emergence of research laboratories in the dyestuff industry, 1870-1900", The British Journal for the History of Science 25 (1992) 91-111

Howell, John D., Technology in the Hospital (Baltimore 1995)

Huhle-Kreutzer, Gabriele, Die Entwicklung arzneilicher Produktionsstätten aus Apothekenlaboratorien (Stuttgart 1989)

Jackson, Charles, Food and Drug Legislation in the New Deal (Princeton 1970)

Kibleur, Pascale, «Le rôle des prescriptions médicamenteuses dans la société française du XVIII ${ }^{\mathrm{e}}$ siècle», Histoire, Economie, Société 3 (2001) 321-337

Lesch, John, "Chemistry and biomedicine in an industrial setting: the invention of the sulfa drugs", in: S. H. Mauskopf (ed.), Chemical Sciences in the Modern World (Philadelphia 1993) $158-215$

- (ed.), The German Chemical Industry in the Twentieth Century (Dordrecht 2000)

- The First Miracle Drug. How the Sulfa Drugs Transformed Medicine (Oxford 2007)

Liebenau, Jonathan, Medical Science and Medical Industry. The Formation of the American Pharmaceutical Industry (London 1987)

- "Paul Ehrlich as a commercial scientist and research administrator", Medical History 34 (1990) 65-78

- /G.J.Higby/E.C. Stroud (eds), Pill Pedlers: essays on the history of the pharmaceutical industry (Madison 1990)

Macfarlane, Gwynn, Howard Florey: the making of a great scientist (Oxford 1979)

- Fleming: l'homme et le mythe (Paris 1984)

Marks, Harry, "Cortisone, 1949: a year in the political life of a drug", Bulletin of the History of Medicine 66 (1992) 419-439

- The Progress of Experiment. Science and therapeutic reform in the United States, 1900-1990 (Cambridge 1997)

Marks, Lara, Sexual Chemistry. A history of the contraceptive pill (New Haven 2001)

Neushul, Peter, "Science, government and the mass production of penicillin", Journal of the History of Medicine and Allied Sciences 48 (1993) 371-395

Oudshoorn, Nelly, Beyond the Natural Body. An archeology of sex hormones (London 1994)

Parascandola, John, "Industrial Research comes of age: the American pharmaceutical industry, 1920-1940", Pharmacy in History 27 (1985) 12-21

Pickstone, John, Ways of Knowing (Manchester 2000)

Pignarre, Philippe, Le grand secret de l'industrie pharmaceutique (Paris 2003)

Plumpe, Gottfried, Die I.G. Farbenindustrie AG: Wirtschaft, Technik und Politik, 1904-1945 (Berlin 1990)

Prüll, Carl R., "Ehrlich and the receptor concept", Medical History 47 (2003) 332-356

Quirke, Viviane, Experiments in Collaboration. The Changing Relationship Between Scientists and Pharmaceutical Companies in Britain and France, 1935-1965, PhD Thesis (Oxford 1999)

- "War and change in the pharmaceutical industry: a comparative study of Britain and France", Entreprises et Histoire 36 (2004) 64-83

- "Making British cortisone: Glaxo and the development of corticosteroids in Britain in the 1950s-1960s", Studies History and Philosophy of the Biological and the Biomedical Sciences 36 (2005) 645-674

Ramsey, Matthew, "Academic medicine and medical industrialism: the regulation of secret remedies in 19th century France", in: Ann La Berge/Mordechai Feingold (eds), French Medical Culture in the 19th Century (Amsterdam 1994) 25-78

Rasmussen, Anne, «Les enjeux d'une histoire des formes pharmaceutiques. La galénique, l'officine et l'industrie $\left(\mathrm{XIX}^{\mathrm{e}}\right.$-début XX $\mathrm{XX}^{\mathrm{e}}$ siècle)», Entreprises et Histoire 36 (2004) 22-45

- «Préparer, produire et présenter des agents thérapeutiques. Histoire de l'objet médicament», dans: Christian Bonah/Anne Rasmussen (éds), Histoire et médicament aux $19^{e}$ et $20^{e}$ siècles (Strasbourg 2005) 159-189 
Rasmussen, Nicolas, "The moral economy of the drug company - medical scientist collaboration in interwar America", Social Studies of Science 34 (2004) 161-185

- "The drug industry and clinical research in interwar America: three types of physician collaborator", Bulletin of the History of Medicine 79 (2005) 50-80

Reader, William J., A History of Imperial Chemical Industries (Oxford 1975)

Reiser, Stanley J., Medicine and the Reign of Technology (Cambridge 1978)

Ruffat, Michelle, «La recherche historique sur l'industrie pharmaceutique en France et à l'étranger», Revue d'Histoire de la Pharmacie 42 (1995) 187-194

- 175 ans d'industrie pharmaceutique française. Histoire de Synthélabo (Paris 1996)

Simon, Jonathan, Chemistry, Pharmacy and Revolution in France, 1777-1809 (Aldershot 2005)

Sinding, Christiane, "Making the unit of insulin: standards, clinical works and industry, 1920-1925", Bulletin of the History of Medicine 76 (2002) 231-270

Slinn, Judy, A History of May \& Baker, 1834-1984 (Cambridge 1984)

Swann, John, Academic Scientists and the Pharmaceutical Industry: cooperative research in 20th century America (Baltimore 1988)

Swazey, Judith, Chlorpromazin in Psychiatry: a Study of Therapeutic Innovation (Cambridge M.A. 1974)

Tanner, Jakob, "The Swiss pharmaceutical industry. The impact of intellectual property rights and trust in the laboratory, 1907-1939", in: Anthony Travis (ed.), Determinants in the Evolution of the European Chemical Industry, 1900-1939 (Dordrecht 1998) 257-272

Temin, Paul, Taking your medicine. Drug regulation in the United States (Cambridge 1980)

Travis, Anthony, "Science as receptor of technology: Paul Ehrlich and the synthetic dyestuffs industry", Science in Context 3 (1989) 383-408

Urfalino, Philippe, Le grand méchant loup pharmaceutique (Paris 2005)

Weatherall, Miles, In Search of a Cure: a History of Pharmaceutical Discovery (Oxford 1990)

Wimmer, Wolfgang, «Wir haben fast immer was Neues». Gesundheitswesen und Innovationen der Pharma-Industrie in Deutschland, 1880-1935 (Berlin 1994)

Young, John H., The Medical Messiahs: a Social History of Health Quackery in 20th century America (Princeton 1967)

- Pure Food: Securing the Food and Drug Act of 1906 (Princeton 1989) 